\section{A Unified Approach to Variable Voltage Scheduling for Nonideal DVS Processors}

\author{
Bren C. Mochocki, Xiaobo Sharon Hu, and Gang Quan
}

\begin{abstract}
Voltage scheduling is an essential technique used to exploit the benefit of dynamic voltage-scaling processors. Though extensive research exists in this area, current processor limitations such as time and energy transition overhead and voltage-level discretization are often dismissed as insignificant. We show that for hard real-time applications, disregarding these details can lead to suboptimal or even invalid results. We propose two algorithms to account for these limitations. The first is a greedy approach, while the second is more complex, but can significantly reduce the system's energy consumption. Through experimental results on both real and randomly generated systems, we show the effectiveness of both algorithms and explore what conditions make it beneficial to use the complex algorithm over the basic one.
\end{abstract}

Index Terms-Embedded systems, low-power design, power minimization, scheduling, simulation.

\section{INTRODUCTION}

The demand for mobile and pervasive computing devices has made low-power/energy computing a critical technology. One of the most effective ways to reduce energy consumption in CMOS processors is dynamic voltage scaling (DVS), i.e., dynamically varying a processor's supply voltage and clock frequency simultaneously. Various DVS processors are commercially available, including Intel's XScale [1], AMD's Mobile Athlon [2], and Transmeta's Crusoe processor [3]. Several research groups have also developed their own DVS systems. For example, Burd and Brodersen implemented a DVS system using the ARM8 core [4], while Pouwelse et al. constructed a similar system using the SA-1100 [5]. Ideally, a DVS processor would operate at any voltage within a specific range and switch from one voltage to another instantaneously. However, due to physical limitations, DVS processors always incur both time and energy overhead during a voltage transition. Furthermore, all commercially available DVS processors today can only operate at discrete voltage levels.

To maximally exploit the benefits of a DVS processor, voltage scheduling, the selection of voltage levels and operating frequencies, is indispensable. A large number of research results have been published on voltage scheduling for DVS processors. These results differ in many aspects, such as the type of applications (e.g., real-time or nonrealtime) considered, the type of systems (e.g., single or multiple processors) used, the location (intratask versus intertask), where a voltage change is allowed, the execution style (e.g., on-line or off-line) of the voltage-scheduling algorithms, etc. It is not difficult to see that nonideal properties of DVS processors, such as discrete voltage levels and transition overhead, can effect voltage-scheduling results and deserves careful study.

In this paper, we focus on voltage scheduling for a set of real-time jobs executed by a single DVS processor. Many embedded applications can be described by such a model. In particular, we study off-

Manuscript received July 14, 2003; revised December 22, 2003 and March 18,2004 . This work was supported in part by the National Science Foundation under grant MIP-9701416, Grant CCR-9988468, and Grant CCR02-08992. This paper was recommended by Associate Editor M. F. Jacome.

B. C. Mochocki and X. S. Hu are with the Computer Science and Engineering Department, University of Notre Dame, Notre Dame, IN 46556 USA (e-mail: bmochock@cse.nd.edu; shu@cse.nd.edu).

G. Quan is with the Computer Science and Engineering Department, University of South Carolina, Columbia, SC 29208 USA (e-mail: gquan@cse.sc.edu). Digital Object Identifier 10.1109/TCAD.2004.833602 line, interjob voltage scheduling where the real-time jobs are executed according to the preemptive earliest deadline first (EDF) scheduling scheme [6]. Preemptive EDF is an optimal scheduling algorithm and has been adopted by many real-time systems [7]. Interjob (or intertask) voltage scheduling is realized by the operation system, which is less intrusive and more portable for a given application. Off-line scheduling does not compete for resources with the actual application and hence can afford to use more sophisticated algorithms. Though off-line scheduling cannot handle dynamic variations, it can often be used as a complement to on-line approaches. The uniqueness of our work is that the impact of practical limitations of DVS processors is analyzed in detail and carefully accounted for during the development of our algorithms.

\section{A. Related Work}

Substantial research exists for scheduling real-time applications on DVS processors, e.g., [8]-[14], some of which have considered off-line, interjob voltage scheduling for preemptive EDF based real-time systems, e.g., [12], [14], [15]. However, a limited amount of work has examined voltage scheduling in the presence of practical limitations. Some of these consider only discrete voltage levels. For example, Chandrasena et al. [16] introduce a rate-selection algorithm for a DVS processor with limited voltage levels, but the algorithm provides no deadline guarantee for the tasks. In [17], Lee et al. consider discrete voltage levels in dynamic (i.e., on-line) voltage scheduling for periodic tasks. Their method, called time slicing, requires that tasks be divided into subtasks (or slots), which is not always possible. Even if the division is possible, preemption is not allowed within a subtask, so this method cannot be applied to the preemptive scheduling problem we address here.

Kwon et al. give an optimal intratask scheduling algorithm under the preemptive EDF scheme to match a discrete set of voltage levels [18]. Swaminathan and Chakrabarty also introduce a method to optimally schedule job sets off-line, given a discrete set of voltage levels. Their method uses network flow techniques to assign a single valid voltage level to each job [15]. Saewong and Rajkumar give an in-depth analysis of the ideal placement of a set of discrete voltage levels for a DVS processor, and conclude that the energy increase due to voltage/frequency quantization is inversely proportional to the number of levels [19]. Since the above approaches ignore transition overhead, they tend to introduce more transitions in order to better match discrete voltage levels, which further exasperate the impact of transition overhead.

A number of researchers have studied voltage scheduling when transition overhead is not negligible. Manzak et al. [20] address the transition-time overhead by linearly increasing the total required execution time or decreasing the processor utilization. Such adjustments may lead to either a deadline miss or an overly pessimistic design. In [21], Hong et al. present a heuristic algorithm that accounts for transition overhead during static scheduling, but assumes both the availability of any voltage level and continuous execution of instructions during a transition. Neither assumption is true for most DVS systems [1]-[3], [5]. AbouGhazaleh et al. propose an intratask voltage-scheduling method that accounts for transition overhead. Their method, however, requires both compiler support and that the source code be engineered to give voltage selection "hints" to the operating system [22]. Hsu and Kremer also present a compiler-driven DVS algorithm, but hard deadlines are not guaranteed [23]. Saewong and Rajkumar account for large time overheads using a method called Sys-Clock, that selects the minimum constant speed that will finish all jobs by their deadlines; essentially avoiding transition overhead by having no transitions at all [19]. Zhang and Chakrabarty consider all three limitations when scheduling voltage levels and checkpoint times 
for fault-tolerant hard real-time systems with periodic tasks [24]. They schedule at most one speed per task, which is coarser than the job-level assignment we consider. Also, they assume that each task can meet all deadlines when running at the smallest processor speed if no faults are present. This assumption will completely eliminate the benefit of DVS for the fault-free environment presented here.

\section{B. Our Contributions}

In this paper, we present several observations regarding the impact of transition overhead on executing real-time jobs according to the preemptive EDF scheme. These observations show that transition time overhead can cause deadline misses as well as a significant increase in energy consumption if not handled carefully. A basic algorithm is devised to guarantee that no deadline violations occur in the presence of transition time overhead. Building on the basic algorithm, we developed an algorithm that considers both transition time and energy overheads as well as discrete voltage levels. A large number of experiments have been conducted for both real-world and randomly generated examples to demonstrate the effectiveness of our algorithms. Careful analysis of the experimental results help us to draw several conclusions regarding the nonideal properties of DVS processors and the performance of our proposed algorithms.

The remainder of this paper is organized as follows. Section II summarizes the relevant background material, including system models and motivation examples. Section III describes our algorithms that handle DVS processor limitations. Section IV presents the experimental results and Section V concludes the paper.

\section{PReliminaries}

\section{A. System Model}

We consider real-time applications consisting of a set of independent jobs, $\mathcal{J}=\left\{J_{1} \ldots J_{n}\right\}$ with each job $J_{i} \in \mathcal{J}$ having a release time $r_{i}$, a deadline $d_{i}$, and worst-case execution cycles $c_{i}$. The job set is to be executed on a DVS processor whose power consumption is a convex function of the processor speed (frequency) [25]. The convexity assumption holds so long as the switching power is one of the main contributors of the total power, which is the case for current and near-future CMOS devices [26].

The DVS processor can operate at a finite set of supply voltage levels $\mathcal{V}=\left\{V_{1}, \ldots, V_{\max }\right\}$, each with an associated speed. To simplify the discussion, we normalize the processor speeds by $S_{\max }$, the speed corresponding to $V_{\max }$, giving $\mathcal{S}=\left\{S_{1}, \ldots, 1\right\}$.

Changing from one voltage level to another takes a fixed amount of time, referred to as the transition interval (denoted as $\Delta t$ ), and consumes a variable amount of transition energy (denoted as $\Delta E$ ). The transition interval length for a DVS processor alone is usually on the order of 10 to $100 \mu \mathrm{s}$ [2], [4], [5]. However, when considering synchronizing with other components in a system, the length can be on the order of milliseconds [19], [27]. Transition energy includes both the energy consumed by the $\mathrm{dc} / \mathrm{dc}$ converter and the CPU. No instructions are executed during a transition.

The above DVS processor model captures the main properties of most commercial DVS processors [2], [5]. A variable length-transition interval (e.g., the one described in [4]) can be approximated by a fixedlength interval equal to the maximum switching time. For processors that do not block instructions during a transition (e.g., [4]), a schedule that assumes blocking during a transition can be pessimistic, but it will guarantee a valid voltage schedule. As in most DVS work, we assume that each job consumes an equal amount of energy per cycle at a given speed, which is a valid assumption for many applications.

We introduce some notation below which will be used throughout the paper. We denote a time interval by $T=\left[t_{s}, t_{f}\right]$ and the interval length by $|T|=t_{f}-t_{s}$. The intensity, $s(T)$, of an interval $T$ is the minimum processor speed required to finish jobs released inside the interval on or before $t_{f}$. It can be readily verified that

$$
s(T)=\frac{\sum_{J_{k} \in \mathcal{J}} c_{k}}{|T|}, \quad r_{k} \geq t_{s} ; \quad d_{k} \leq t_{f}
$$

For a given job set $\mathcal{J}$, the critical interval is defined as the interval with the highest speed. The algorithms we describe in this paper operate by iteratively identifying and scheduling each critical interval. The critical interval found at iteration $i$ is denoted by $T_{i}=\left[t_{s}(i), t_{f}(i)\right]$ and executes a set of jobs $\mathcal{J}_{i} \in \mathcal{J}$ at a speed $s_{i}$. Two critical intervals $T_{i}$ and $T_{j}$ with $i>j$ are said to be adjacent if one of the following conditions is true: 1) $t_{f}(j) \leq t_{s}(i)$ and there are no other critical intervals or unscheduled jobs in $\left[t_{f}(j), t_{s}(i)\right]$, i.e., $T_{j}$ is to the left of $T_{i} ; 2$ ) $t_{f}(i) \leq t_{s}(j)$ and there are no other critical intervals or unscheduled jobs in $\left[t_{f}(i), t_{s}(j)\right]$, i.e., $T_{j}$ is to the right of $T_{i}$; or 3$) t_{s}(i) \leq t_{s}(j)$ and $t_{f}(j) \leq t_{f}(i)$, i.e., $T_{i}$ contains $T_{j}$. For the rest of this paper, we may omit the word transition when referring to transition time/energy overhead.

\section{B. Low-Power Earliest Deadline First (LPEDF)}

We briefly review the voltage scheduling algorithm LPEDF, presented in [12], as it is referenced throughout the paper. LPEDF is rephrased in Algorithm 1.

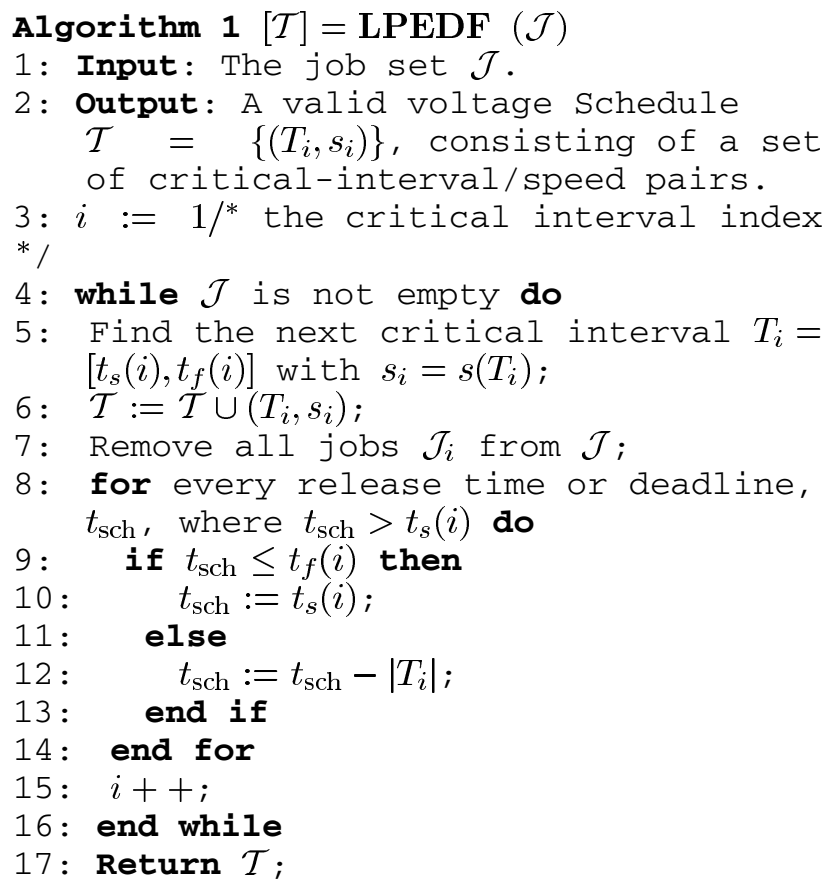

LPEDF finds off-line an optimal voltage schedule for a set of independent tasks executed according to the preemptive EDF policy. It assumes an ideal DVS processor without transition overhead. The general idea is to iteratively identify (line 5), schedule (lines 6 and 7) and remove (lines 8-14) each critical interval. Lines 8-14 essentially "squeeze" the critical interval to a single time point at $t_{s}(i)$ by reducing all release times or deadlines inside $T_{i}$ to $t_{s}(i)$ and then reducing all release times or deadlines after $t_{f}(i)$ by $\left|T_{i}\right|$. For the rest of the paper, when we refer to squeezing an interval, we mean performing a similar operation.

Algorithm 1 is greedy in the sense that it always picks the critical interval to schedule first. Consequently, the intervals are identified according to a monotonically nonincreasing order of their associated speeds. Due to the convexity of the power function, this monotonicity 

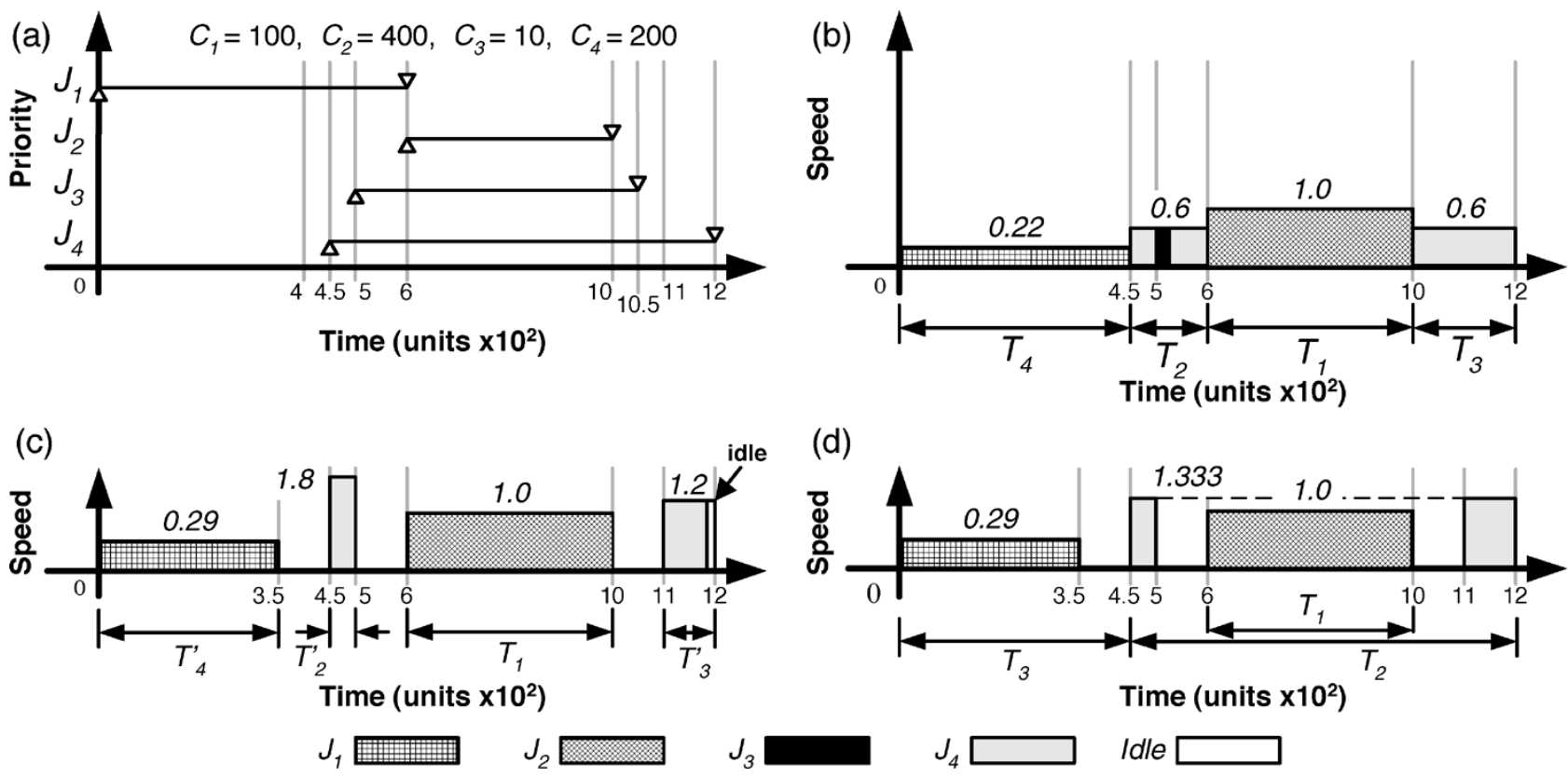

Fig. 1. (a) Example set of jobs. (b) Optimal voltage schedule using LPEDF. (c) LPEDF schedule, modified by inserting $\Delta t=100$ at each transition, and then rescaling $T_{2}, T_{3}$ and $T_{4}$ to maintain the same executed workload. (d) Schedule produced by M-LPEDF.

property (summarized formally in Lemma 1) is beneficial when constructing voltage schedules. For the proof of Lemma 1 and more details on LPEDF, we direct the readers to [12].

Lemma 1: Critical intervals found by successive iterations of LPEDF are monotonically nonincreasing in intensity, that is, $s_{i} \geq s_{j}$ if $i \leq j$.

\section{Motivational Example}

To illustrate the impact of ignoring transition overhead, we present the following example. Consider the job set in Fig. 1(a), which contains four jobs (where $\triangle$ represents a job release time and $\nabla$ a job deadline). The optimal voltage schedule by LPEDF, assuming both $\Delta t$ and $\Delta E$ are zero, is given in Fig. 1(b). Suppose the same set of jobs is scheduled on a DVS processor with $\Delta t=100$.

A straightforward approach to include time overhead is to: 1) insert a transition interval at each speed change and 2) rescale the speed of the interval with the lower speed to ensure that the same amount of work is completed. The resulting schedule is shown in Fig. 1(c). The speed of $T_{2}^{\prime}$, the modified interval $T_{2}$, was calculated using

$$
s_{2}^{\prime}=s_{2} \frac{\left|T_{2}\right|}{\left|T_{2}^{\prime}\right|}=0.6 \frac{(6-4.5)}{(5-4.5)}=1.8 .
$$

The schedule in Fig. 1(c) is invalid. First, $s_{2}^{\prime}$ and $s_{3}^{\prime}$ are now higher than $s_{1}$, which we refer to as a monotonicity violation because it violates Lemma 1 . Note that $s_{2}^{\prime}$ and $s_{3}^{\prime}$ also surpass the normalized maximum of 1, referred to as overshoot. Second, $J_{3}$ will never be executed. Notice that $r_{3}=500$ and $d_{3}=1050$, but no cycles can be executed during the transition intervals $[500,600]$ and $[1000,1100]$ and the critical interval $[600,1000]$ is completely utilized by $J_{2}$. We refer to this problem as an execution violation. Finally, notice that there is a sliver of idle time in the interval $[1192,1200]$. When $T_{2}^{\prime}$ was formed from $T_{2}$ to accommodate the transition interval, it still included the execution cycles of $J_{3}$, even though $T_{2}^{\prime}$ is outside $\left[r_{3}, d_{3}\right]$. The extra time was used by $J_{4}$ during $T_{2}^{\prime}$, so the idle part of $T_{3}^{\prime}$ is not required to complete $J_{4}$. Because (2) fails to take into account release times and deadlines when rescaling the speed, it can result in schedules that require jobs to execute before they are released or after their deadlines.
A more sophisticated modification to LPEDF follows. Instead of compressing just the critical interval $T_{i}=\left[t_{s}(i), t_{f}(i)\right]$ down to a single time point, compress the interval $\left[t_{s}(i)-\Delta t, t_{f}(i)+\Delta t\right]$ and adjust adjacent jobs accordingly. We refer to this approach as M-LPEDF. With M-LPEDF, a job $J_{i}$ will never be scheduled for execution outside $\left[r_{i}, d_{i}\right]$. Applying M-LPEDF to the system in Fig. 1(a), we obtain the schedule shown in Fig. 1(d). Unfortunately, the schedule in Fig. 1(d) still has monotonicity and execution violations since $s_{2}>s_{1}$ and $J_{3}$ is contained in the transition intervals about $T_{1}$. Furthermore, the schedule is still not feasible due to the overshoot of $T_{2}$. The next section presents two algorithms that guarantee valid schedules.

\section{AlgORITHMS FOR DVS PROCESSORS WITH PRACTICAL LIMITATIONS}

In this section, we first present a scheduling algorithm that can guarantee the feasibility of real-time jobs in the presence of time overhead. We then introduce an enhanced algorithm to improve the energy efficiency of this algorithm and incorporate discrete speeds and transition energy overhead simultaneously.

\section{A. Basic Algorithm for Time Overhead}

Regarding monotonicity violations induced by M-LPEDF, we have observed that any critical interval that violates Lemma 1 must be adjacent to the critical interval identified in the previous iteration. (Note that generally critical intervals found in successive iterations are not necessarily adjacent to one another). This observation is stated formally in Lemma 2.

Lemma 2: Let $T_{i-1}$ and $T_{i}$ be two critical intervals obtained by M-LPEDF. If $s_{i-1}<s_{i}$, then $T_{i-1}$ is adjacent to $T_{i}$.

Proof: To schedule $T_{i-1}=\left[t_{s}(i-1), t_{f}(i-1)\right]$, M-LPEDF compresses $T_{i-1}^{\prime}=\left[t_{s}(i-1)-\Delta t, t_{f}(i-1)+\Delta t\right]$. This modification does not change the workload distribution of jobs outside $T_{i-1}^{\prime}$. Only intervals adjacent to or overlapping $T_{i-1}^{\prime}$ are altered; shortened by up to $\Delta t$ time or by exactly $2 \Delta t$ time if $T_{i}$ contains $T_{i-1}^{\prime}$. Thus, only intervals adjacent to $T_{i-1}$ may experience an increase in intensity in the next iteration. 
The key observation leading to Lemma 2 is that a monotonicity violation occurs when the allotted time intervals for executing some jobs are shortened due to the addition of the transition intervals of the previous critical interval. The result is that these jobs require a higher speed in order to meet their deadlines. To remove such a violation, we could simply eliminate the transitions by merging jobs in the violation interval with those in the previously identified critical interval. However, we need to be sure that such a merge will not lead to any deadline misses. Lemma 3 below provides this guarantee.

Lemma 3: Let $T_{i-1}$ and $T_{i}$ be two critical intervals obtained by M-LPEDF with $s_{i-1}<s_{i}$. The minimum speed at which every job $J_{k} \in\left(\mathcal{J}_{i-1} \cup \mathcal{J}_{i}\right)$ can execute and still meet its deadline is $s_{i-1}$.

Proof: According to Lemma 2, $T_{i-1}$ and $T_{i}$ must be adjacent. Therefore, if the speed $s_{i-1}$ is applied to both of these intervals, no voltage transition occurs. According to Lemma $1, s_{i-1}$ is the minimum speed required to guarantee the deadlines for jobs in $\mathcal{J}_{i-1}$ and is greater than the speed needed to guarantee the deadlines for jobs in $\mathcal{J}_{i}$, when no time overhead is present. Therefore, $s_{i-1}$ is the minimum speed required for every job in $\left(\mathcal{J}_{i-1} \cup \mathcal{J}_{i}\right)$ to meet its deadline.

Based on Lemmas 2 and 3, we can keep track of monotonicity violations and remove them whenever they occur. In fact, these lemmas also help eliminate execution violations. Observe that an execution violation is just a special case of a monotonicity violation, where the speed required by the violation interval is $\infty$. For instance, in Fig. 1(d), after applying M-LPEDF at the first iteration, $T_{1}$ is identified and [600$100,1000+100]$ is compressed. This results in the modification of $r_{3}$ and $d_{3}$ where $r_{3}^{\prime}=d_{3}^{\prime}=500$. During the next scheduling iteration, the degenerate interval $[500,500]$ is identified since a speed of $\infty$ is needed to finish $J_{3}$ in zero time. Consequently, the new critical interval causes a monotonicity violation.

Building upon the above lemmas and observations, we propose Algorithm 2, called time overhead earliest deadline first (TOEDF), which eliminates both monotonicity and execution violations as they occur, thus producing a feasible schedule with a time overhead of arbitrary size.

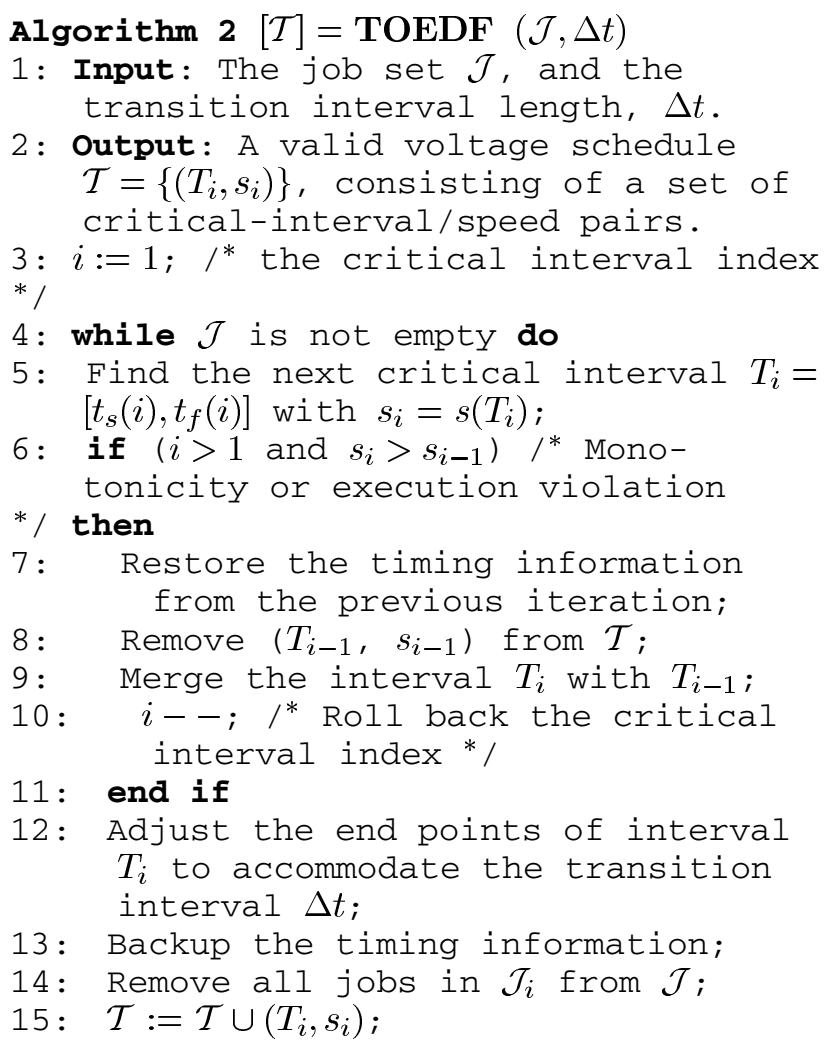

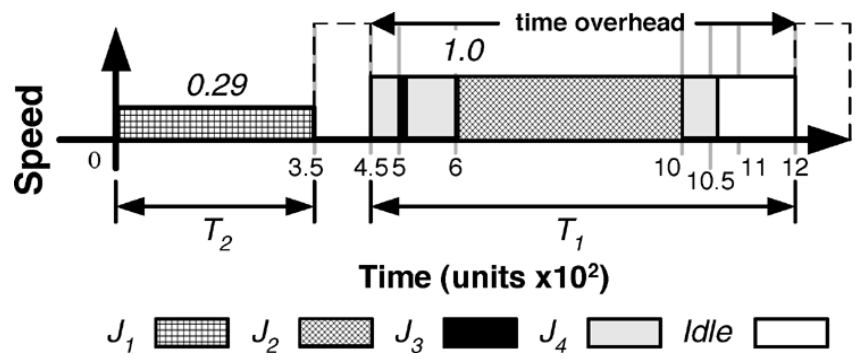

Fig. 2. Job set from Fig. 1(a), scheduled by TOEDF.

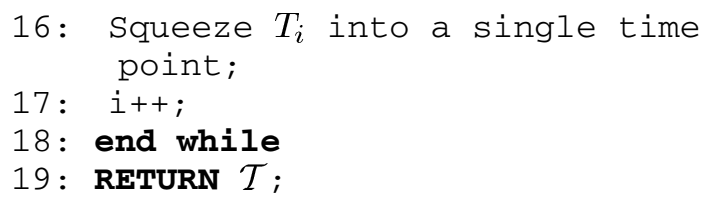

In TOEDF, when a monotonicity or execution violation is encountered (line 6), $T_{i-1}$ is extended to include all jobs scheduled in $T_{i}$ (line 9). "Squeezing" a critical interval changes the timing parameters of some jobs (line 16). The parameters are required if $T_{i+1}$ causes a violation, so we save these parameters (line 13). After $T_{i}$ is identified, we may need to extend it like M-LPEDF to incorporate $\Delta t$ (line 12). One must be particularly careful during this step. For example, if an end point of $T_{i}$ is within $\Delta t$ of any previous interval (say $T_{j}$ ) in $\mathcal{T}$, we should not extend $T_{i}$ so that it overlaps $T_{j}$. This will prevent TOEDF from including more time overhead than necessary. (This can be done by maintaining a proper data structure when implementing the algorithm. We omit the details.) Theorem 1 below summarizes the correctness and complexity of Algorithm 2. The proof follows directly from Lemmas 2 and 3 and is thus omitted. A detailed proof can be found in [28].

Theorem 1: Algorithm 2 always produces a valid voltage schedule in $O\left(n^{3}\right)$ time, given an initially schedulable job set.

In what follows, we apply Algorithm 2 to the job set in Fig. 1(a) and discuss more details of this algorithm. During the first iteration of Algorithm 2, the critical interval $[600,1000]$ with $s_{1}=1$ is identified. The interval is extended to $[500,1100]$ (with $\Delta t=100$ ) and then $\left(T_{1}, s_{1}\right)$ is inserted into $\mathcal{T}$. During the second iteration, $J_{3}$ causes an execution violation. Therefore, $\left(T_{1}, s_{1}\right)$ is removed from $\mathcal{T}$ and $J_{3}$ is merged with $J_{2} \in \mathcal{J}_{1}$. The new $T_{1}$ is expanded to [500, 1050] to reflect the earliest release time and the latest deadline of both jobs $J_{2}$ and $J_{3}$, and is further expended to $[400,1150]$ to accommodate time overhead. $T_{1}$ keeps $s_{1}$ from the previous iteration and $\left(T_{1}, s_{1}\right)$ is once again inserted into $\mathcal{T}$.

In the third iteration, $J_{4}$ causes a monotonicity violation. Therefore, $T_{1}=[400,1150]$ is extended to $[350,1300]$. Finally, during the fourth iteration another critical interval, $T_{2}=[0,350]$ is identified with $s_{2}=$ $s\left(T_{2}\right)=100 / 350=0.29$. Note that $t_{f}(2)=t_{s}(1)=350 . T_{1}$ already includes a transition interval from 350 to 450 , so $\left(T_{2}=[0,350], s_{2}\right)$ is inserted into $\mathcal{T}$ (we assume no transition at time 0 .) The resulting schedule is depicted in Fig. 2. One can readily verify that this schedule indeed guarantees the schedulability of all the jobs.

\section{B. Improving the Basic Algorithm}

Unnecessary energy may be wasted when using TOEDF since, in order to eliminate violations, we use "higher-than-necessary" speeds for some intervals. Although removing violations is critical for guaranteeing the feasibility of a voltage schedule, one should strive to shorten the intervals that demand higher speeds to reduce energy consumption. For example, when applying TOEDF to the job set in Fig. 1(a), the 
processor speed for the interval $[400,1200]$ is 1.0 . However, one can readily verify that using the processor speed of 1.0 during the interval $[590,1200]$ can also guarantee the schedulability of the jobs scheduled in that interval, i.e., $J_{2}-J_{4}$. Energy is saved in two ways: first, the interval length that demands the speed of 1 is reduced; second, extra time is available for any remaining jobs, such as $J_{1}$, which can be used to further reduce their required speed. In this case, $J_{1}$ can be executed at $100 / 490=0.2$, instead of 0.29 like the schedule in Fig. 2. Therefore, if we have to use a higher-than-necessary speed, we should restrict the usage of this speed to as short of an interval as possible. The problem can be formulated as follows. Given a set of jobs, $\mathcal{J}$, and a constant speed, $s^{*}$, where $s^{*}$ is a constant speed no less than the minimum speed required to meet all deadlines of $\mathcal{J}$, find the shortest interval in which all deadlines of $\mathcal{J}$ are met.

The key to solving this problem is to realize that we really have one degree of freedom, i.e., how long the start of the interval can be delayed. To prevent any deadline misses due to the delayed executions, we must identify the latest start time for the job set, i.e., the latest time at which jobs in $\mathcal{J}$ can begin execution at speed $s^{*}$ and still meet all deadlines.

Lemma 4: The job set $\mathcal{J}$ scheduled by EDF has the latest start time

$$
t_{\mathrm{LS}}=\min \left\{t_{\mathrm{LS}}(i)\left|t_{\mathrm{LS}}(i)=d_{i}-\sum_{J_{k} \in h p\left(J_{i}\right)} \frac{c_{k}}{s^{*}}, i=1 . .\right| \mathcal{J} \mid\right\}
$$

where $h p\left(J_{i}\right)$ is the job set containing jobs with priorities equal to or higher than the priority of $J_{i}$.

Proof: First, we prove that starting at a time later than $t_{\mathrm{LS}}(i)$ causes $J_{i}$ to miss its deadline. Then, we prove that $J_{i}$ does not miss its deadline if execution begins at or before $t_{\mathrm{LS}}(i)$.

1) By EDF, $h p\left(J_{i}\right)$ includes the jobs with deadlines no later than $d_{i}$. To guarantee that all jobs in $h p\left(J_{i}\right)$ meet their deadlines, there must be sufficient time to execute the cycles of these jobs. The total workload of jobs in $h p\left(J_{i}\right)$ is $W=\sum_{J_{k} \in h p\left(J_{i}\right)} c_{k}$, and the total time needed to execute this workload is $W / \mathrm{s}^{*}$. It is trivial to see that starting later than $t_{\mathrm{LS}}(i)$ will not give enough time to finish $W$. The minimum $t_{\mathrm{LS}}(i)$ is the most restrictive latest start time of all jobs in $\mathcal{J}$, so it is the correct choice for $t_{\mathrm{LS}}$.

2) Suppose that beginning execution at $t_{\mathrm{LS}}(i)$ causes $J_{i}$ to miss its deadline. Considering the execution of jobs in $h p\left(J_{i}\right)$, there must be idle time in the interval $\left[t_{\mathrm{LS}}(i), d_{i}\right]$ such that no job in $h p\left(J_{i}\right)$ is executed during this idle time. Beginning execution at any time earlier than $t_{\mathrm{LS}}(i)$ will not alter the job released after the idle interval (including $J_{i}$ based on EDF), so $J_{i}$ must still miss its deadline. This contradicts the assumption that all jobs in $\mathcal{J}$ executing at the speed $s^{*}$ will finish by their deadlines.

Lemma 4 provides a way to find the latest start time $\left(t_{\mathrm{LS}}\right)$ for a given set of jobs and a constant speed $s^{*}$. Then, with a simple planesweeping algorithm we find the earliest finish time $t_{\mathrm{EF}}$ of all jobs in $\mathcal{J}$ based on $t_{\mathrm{LS}}$. Together, $t_{\mathrm{LS}}$ and $t_{\mathrm{EF}}$ form a minimum time interval. We refer to this procedure as Algorithm MININT. To show that MININT indeed produces a minimum-length valid interval, we present Theorem 2. The proof follows from Lemmas 3 and 4 . The complete algorithmic description of MININT and proof of Theorem 2 can be found in [28].

Theorem 2: Given a set of jobs, $\mathcal{J}$, and a constant speed, $s^{*}$, Algorithm MININT finds a minimum length interval $T=\left[t_{s}, t_{f}\right]$ needed to complete every job $J_{k} \in \mathcal{J}$ at the speed $s^{*}$ by its deadline.

MININT can be readily incorporated into TOEDF to improve its energy performance. In TOEDF, when a violation occurs and jobs are merged with those executed in the previous critical interval, we can apply MININT to find the shortest interval within which every job deadline is satisfied. This typically results in increased energy savings.

\section{Discrete Voltage/Frequency Levels}

Until now, we have assumed that the processor speed can be continuously varied. However, current commercial DVS processors [1]-[3] only have a finite number of speeds. This factor must be integrated into voltage-scheduling algorithms to provide a valid and energy-efficient schedule.

One intuitive way to deal with discrete speeds is to round up the required frequency and voltage to some allowed level. Unfortunately, this can be extremely pessimistic and energy inefficient, especially for many commercial processors with only a few voltage levels available [2]. A better approach is proposed in [18] and [29] that can use the two levels immediately above and below the desired voltage/speed value to optimally schedule a single job. Although theoretically optimal, this approach is not practically applicable on real processors because excessive voltage transitions, roughly two per job, are introduced. This, coupled with the omission of time overhead, will cause jobs to miss their deadlines.

To consider both time overhead and discrete speeds simultaneously, we believe that it is more advantageous to incorporate the discrete speed effects into the construction of critical intervals and let it propagate to future critical interval construction. Specifically, after a critical interval is identified in Algorithm 2, its speed is increased to the next available level. Recall that when a higher-than-necessary speed is applied, we can use MININT to find the minimal interval needed for the given job set and speed. However, when increasing the speed to the higher level, it can introduce a significant amount of unused idle time, even after we apply algorithm MININT to find a minimal length interval. A better method to utilize these idle times to save energy is to relax the requirement that all jobs originally found in the critical interval must run at the higher speed (note that Lemma 3 only holds when the speed increase is due to a violation). Therefore, we keep only one of these busy intervals (intervals without idle time) for the final voltage schedule, with the expectation that the rest of the jobs may benefit from the higher-than-necessary speed assignment for this interval and can execute at a lower speed. One problem is how to select which busy interval to keep. A good choice can lead to low computation cost and higher energy efficiency. There are a number of heuristics, such as always selecting the first, the last, the shortest, or the longest busy intervals. Though each of these approaches has its intuitive advantages, none of them dominates the others in our experiments. This is due to the many patterns of job arrival times, deadlines, and execution cycles. Therefore, we simply choose the first busy interval as it is the most computationally convenient. We refer to this procedure as Algorithm DISCRETE. A detailed description of DISCRETE can be found in [28].

\section{Transition-Energy Overhead}

So far, we have ignored energy overhead in our voltage scheduling algorithms. Similar to time overhead, we account for energy overhead while constructing the critical intervals. This allows the effect to propagate throughout the schedule. Our approach works as follows: when a new critical interval, $T_{i}$ is identified, whether or not this critical interval is kept depends on whether or not the energy consumed by adopting its speed is smaller than that consumed by merging it with an adjacent critical interval, $T_{j}$. (The term adjacent is defined in Section II-A). The idea is that if the energy transition overhead is so significant that using different processor speeds for different intervals consumes more energy than using a single speed, we can simply merge adjacent critical intervals by adopting the largest speed among them for every job and then removing the associated voltage transitions. Again, MININT is used to find a minimum-length interval. We refer to this procedure as Algorithm ENERGY_OH and refer the readers to [28] for more details. 


\section{E. Unified Algorithm}

By combining the techniques from Sections III-B to III-D with Algorithm 2, a valid voltage schedule with superior energy savings is produced while accounting for practical limitations of real-world DVS processors, including time and energy overhead and discrete voltage levels. We call this unified algorithm UAEDF (see Algorithm 3).

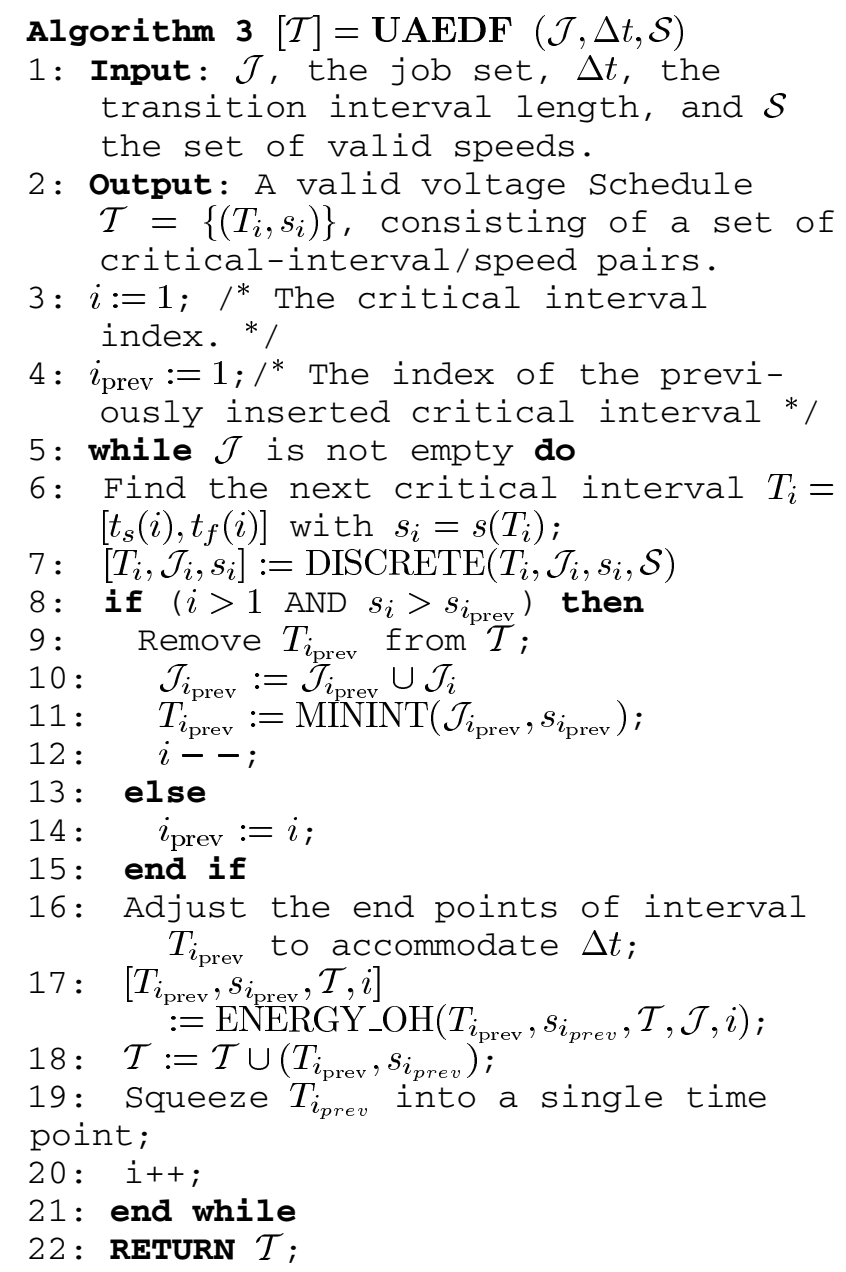

Algorithm 3 follows the same general flow as Algorithm 2. First, it identifies the next critical interval assuming any speed is valid (line 6) and matches the next valid speed using DISCRETE (line 7). Then, it removes monotonicity or execution violations and shortens the critical interval with MININT (lines 9-12). ENERGY_OH is used to minimize the impact of energy overhead (line 17). Finally, as in TOEDF, the newly generated interval is "squeezed" into one single time point (line 19) and one iteration of the algorithm is completed. Theorem 3 guarantees the correctness of Algorithm 3. The proof follows directly from Theorems 1 and 2 and is thus omitted. A detailed proof can be found in [28]).

Theorem 3: Algorithm 3 always produces a valid voltage schedule with a time complexity of $O\left(n^{3}\right)$.

\section{EXPERIMENTAL RESULTS}

In this section, we quantify the impact of transition overhead and discrete voltage levels and evaluate the energy savings of our proposed algorithms with both randomly generated job sets and real-world examples. We compare our algorithms against Sys-Clock, presented in [19], modified to schedule EDF job sets rather than RM task sets. As far as we know, Sys-Clock is the only previous work that can include an arbitrarily large time overhead and still guarantee a valid voltage schedule.

In our experiments, we base our power model on the AMD Mobile Athlon4 DVS processor [2]. The Mobile Athlon4 can run at voltage levels in the range of 1.2-1.4 V with $50 \mathrm{mV}$ steps and corresponding frequencies of $500 \mathrm{MHz}$ to $1 \mathrm{GHz}$ with $100 \mathrm{MHz}$ steps. For experiments with more than five voltage levels, we interpolate these performance points using a second-order polynomial. The minimum speed/voltage for all experiments is $500 \mathrm{MHz}$ at $1.2 \mathrm{~V}$. Power is modeled using the equation $P=C_{\mathrm{SW}} f_{\mathrm{op}} V_{\mathrm{DD}}^{2}$. The value of $C_{\mathrm{SW}}$ is set to $12.75 \mathrm{nF}$, based on information from AMD's datasheet [2]. The CPU power ranges from 9.2 to $25 \mathrm{~W}$. The system includes a low power "sleep" state that the processor can utilize when idle. We assume that it takes $\Delta t / 2$ time to enter the sleep state, and another $\Delta t / 2$ to exit. The power consumed while in the sleep state is $2.4 \mathrm{~W}$.

Time overhead is modeled by a constant value in the range of 0 (no time overhead) to $5 \mathrm{~ms}$. Note that while most DVS processors have a time overhead in the range of tens to hundreds of microseconds, these numbers may be misleading as they ignore synchronization delay with off chip components such as memory, which can be quite large. The Compaq iPAQ, for example, requires $20 \mathrm{~ms}$ to synchronize with its main memory after a frequency switch [27]. Energy overhead is modeled using $\Delta E=\Delta E_{\mathrm{DC}}+\Delta E_{\mathrm{CPU}}$ where $\Delta E_{\mathrm{DC}}$ is the energy consumed by the dc-dc converter, and $\Delta E_{\mathrm{CPU}}$ is the energy consumed by the CPU during a transition. $\Delta E_{\mathrm{DC}}=\eta C_{\mathrm{DD}}\left|V_{\mathrm{DD} 1}-V_{\mathrm{DD} 2}\right|$ with $C_{\mathrm{DD}}=100 \mu \mathrm{F}$ and $\eta=0.9$ as presented by Burd in [30]. $\Delta E_{\mathrm{CPU}}=k \Delta t$ where $k=2.4 \mathrm{~W}$ is the power consumed in the stop-grant state of the AMD processor (entered during a transition) according to [2].

We first construct and test 100 randomly generated sets of 20 jobs each. The jobs are assumed to always require the worst-case execution cycles to complete. Execution cycles and release times are uniformly distributed between $[0,800]$ and $[0,1000] \mu$ s, respectively. The relative deadlines of the jobs are normally distributed with an average of $810 \mu \mathrm{s}$ and a standard deviation of $280 \mu \mathrm{s}$. We then schedule each job set using Sys-Clock, TOEDF, and UAEDF on a processor with $\propto, 14,5$, and 2 discrete voltage levels. The energy from each schedule was normalized against the optimal LPEDF schedule without transition overhead on a continuous processor, i.e., we use LPEDF as the lower bound on energy. The results are summarized in Fig. 3. Next, we apply our algorithms to three real-world examples: 1) a computerized numeric controller (CNC) task set based on the work by Kim et al. in [31]; 2) an Avionics task set based on Locke's work in [32]; and 3) a video phone task set based on based on the work by Shin et al. in [11]. Each task set is first converted to a job set by unrolling the tasks to the set's respective system hyperperiod. These results are given in Figs. 4-6.

From the experimental results, one can immediately conclude that TOEDF and UAEDF always outperform Sys-Clock in terms of energy efficiency. For example, in Fig. 3(b), when the time overhead is $0.5 \mathrm{~ms}$ and the number of voltage levels is 14, TOEDF and UAEDF outperform Sys-Clock by as much as $30 \%$ and $39 \%$ respectively. Moreover, our experiments also show the significant improvement of UAEDF over TOEDF, especially with the increased time overhead and fewer discrete voltage levels. In Fig. 3(b), at low time overhead, i.e., between 0 and $200 \mu \mathrm{s}$, TOEDF and UAEDF are within $2 \%$ of each other. However, when the time overhead is $0.9 \mathrm{~ms}$, UAEDF outperforms TOEDF by around $13 \%$. Also, at two levels [Fig. 3(c)], the difference becomes approximately $25 \%$.

The same conclusion can be drawn from our practical application experiments as shown in Figs. 4-6. Compared to the random job sets, it is interesting to note that the energy consumption does not increase monotonically with time overhead. This is because for a particular job 
(a)

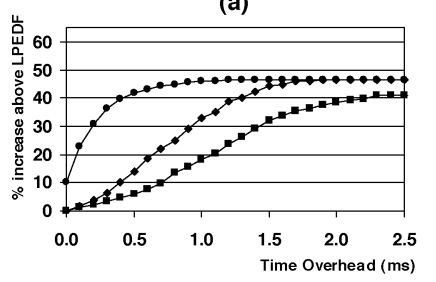

(b)

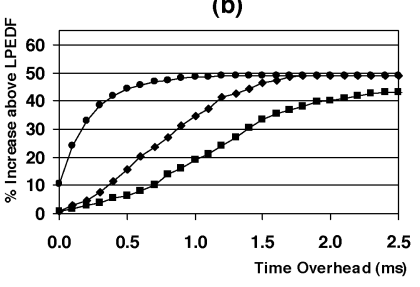

(c)

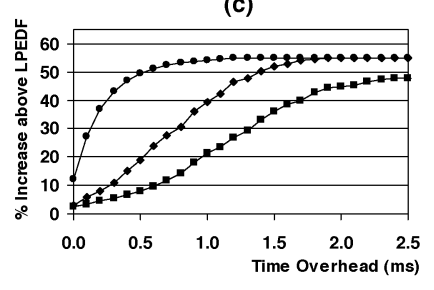

(d)

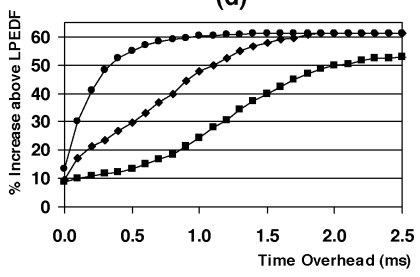

Fig. 3. Average increase in energy above LPEDF for 100 random job sets. (a) Continuous, (b) 14, (c) 5, and (d) 2 voltage levels.

(a)

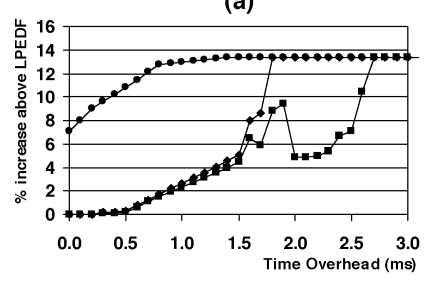

(b)

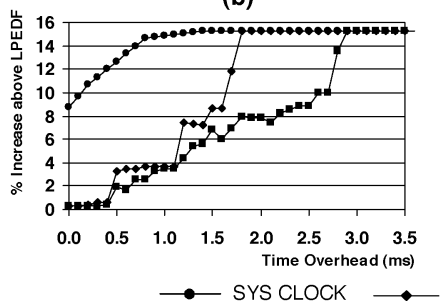

(c)

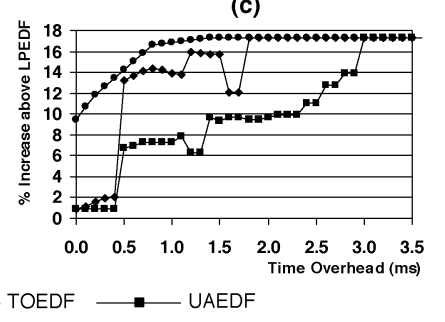

(d)

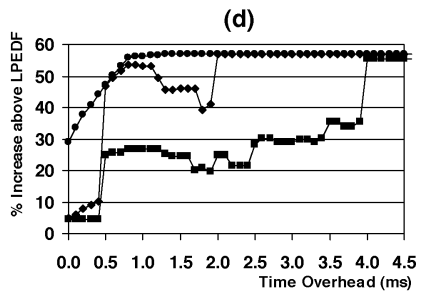

Fig. 4. Increase in energy above LPEDF for the CNC job set. (a) Continuous, (b) 14, (c) 5, and (d) 2 voltage levels.

(a)

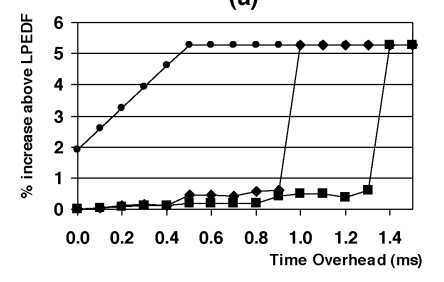

(b)

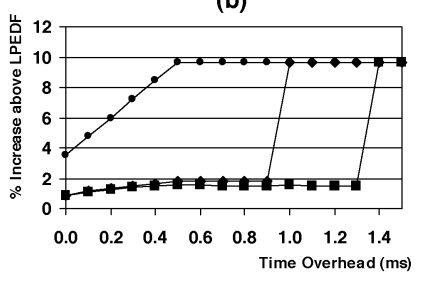

(c)

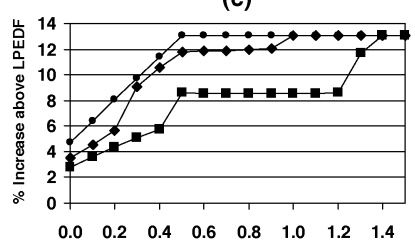

Time Overhead (ms)

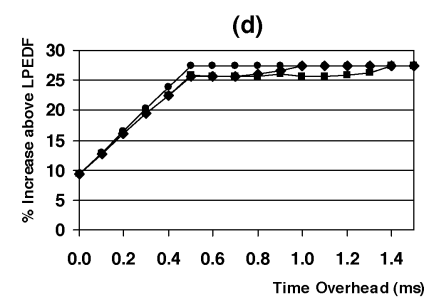

Fig. 5. Increase in energy above LPEDF for the Avionics job set. (a) Continuous voltage levels; (b) 14 voltage levels; (c) 5 voltage levels; (d) 2 voltage levels.

(a)

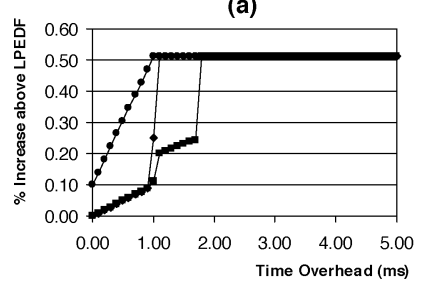

(b)

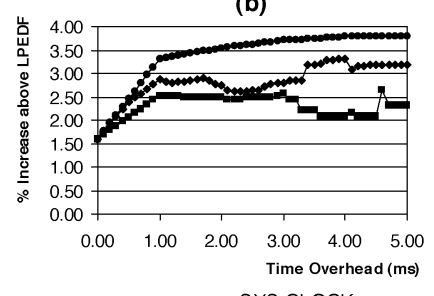

(c)

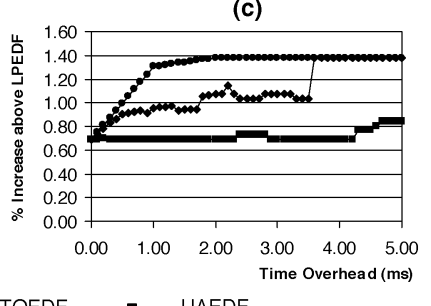

(d)

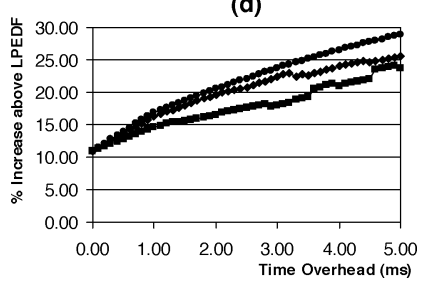

Fig. 6. Increase in energy above LPEDF for the video phone job set. (a) Continuous voltage levels; (b) 14 voltage levels; (c) 5 voltage levels; (d) 2 voltage levels.

set, some values of $\Delta t$ may be "nicer" than others, as the impact of $\Delta t$ depends on the timing parameters of the given jobs.

Finally, we would like to point out an important aspect not illustrated in our figures, i.e., the energy reduction from including and excluding the energy overhead part of UAEDF. In our experiments the difference was minimal (less than $1 \%$ variation). There are three main reasons for this result. First, our algorithms already handle time overhead by reducing the number of transitions which naturally reduces the impact of energy overhead also. Second, the energy overhead that is not directly dependent on $\Delta t$, i.e. that incurred by the dc-dc converter (maximally $\left.(0.9)\left(100 \times 10^{-6}\right)\left|1.4^{2}-1.2^{2}\right|=0.0468 \mathrm{~mJ}\right)$ is less than $1 \%$ of the energy consumed by a $1 \mathrm{~ms}$ interval executing at even the minimum power level $(9.2 \mathrm{~mJ})$. Typically critical intervals are longer than $1 \mathrm{~ms}$. Third, assuming that avoiding a transition to eliminate an instance of energy overhead does save energy, the savings are balanced by the execution of some cycles at a higher speed instead of a lower one. This is particularly true for fewer discrete voltage levels.

\section{SUMMARY}

In this paper, we study the impact that practical limitations of current DVS processors can have on the energy consumed in a hard real-time system. These limitations include time and energy overhead and discrete voltage levels. We have shown through examples and analysis that transition time overhead can cause a theoretically feasible off-line schedule to become invalid if not correctly accounted for during the scheduling process.

We present two algorithms, TOEDF and UAEDF, which always produce valid voltage schedules in the presence of time overhead for a real-time job set theoretically schedulable according to EDF. Our experiments demonstrate that these two algorithms can outperform the previous approach by up to 39\%. TOEDF, built on LPEDF [12], constructs a feasible schedule by addressing monotonicity and execution violations that occur when time overhead is introduced. UAEDF enhances TOEDF by reducing the length of intervals in which a high 
voltage is needed. In addition, it also takes discrete voltages level and energy overhead into consideration, giving UAEDF increased energy saving potential. Our experiments show that, while UAEDF has comparable energy savings to TOEDF when the time overhead is small, it can greatly improve energy performance, up to $25 \%$ when the time overhead becomes significant. This is particularly appealing in the face of rapidly elevated operation frequencies in today's commercial processors.

Currently, the optimality of our algorithms is not guaranteed, so further algorithm development may improve results even more. Also, for the scheduling process to give a practical voltage schedule for an even wider range of systems, we need to account for other implementation details, such as context switching overhead, different transition models and support for other priority schemes, such as fixed-priority scheduling.

\section{REFERENCES}

[1] "The Intel Xscale Microarchitecture," Intel Corporation, Tech. Rep., 2000.

[2] "Mobile AMD Athlon 4 processor model 6 CPGA data sheet rev:e," Advanced Micro Devices, Tech. Rep. 24319, Nov. 2001.

[3] M. Fleischmann, "Longrun power management: Dynamic power management for Crusoe processors," Advanced Micro Devices, Tech. Rep. 24319, Jan. 2001.

[4] T. D. Burd and R. W. Brodersen, "Design issues for dynamic voltage scaling," in Proc. Int. Symp. Low-Power Electron. Design, July 2000, pp. 9-14.

[5] J. Pouwelse, K. Langendoen, and H. Sips, "Dynamic voltage scaling on a low-power microprocessor," in Proc. 7th Annu. Int. Conf. Mobile Comput. Networking, July 2001, pp. 251-259.

[6] C. L. Liu and J. W. Layland, "Scheduling algorithms for multiprogramming in a hard-real-time environment," J. ACM, vol. 20, no. 1, pp. 46-61, Jan. 1973

[7] J. W. S. Liu, Real-Time Systems. Englewood Cliffs, NJ: Prentice Hall, 2000.

[8] W. Kim, J. Kim, and S. L. Min, "Dynamic voltage scaling algorithm for dynamic-priority hard real-time systems using slack time analysis," in Proc. Design, Automation, Test Eur. Conf. Exhibit., Mar. 2002, pp. 788-794.

[9] T. Okuma, T. Ishihara, and H. Yasuura, "Software energy reduction techniques for variable-voltage processors," Design Test Comput., vol. 18, no. 2, pp. 31-41, 2001.

[10] G. Quan and X. S. Hu, "Energy efficient fixed-priority scheduling for real-time systems on variable voltage processors," in Proc. Design Automation Conf., June 2001, pp. 828-833.

[11] D. Shin, S. Lee, and J. Kim, "Intra-task voltage scheduling for low-energy hard real-time applications," Design Test Comput., vol. 18, no. 2, pp. 20-30, 2001.

[12] F. Yao, A. Demers, and S. Shenker, "A scheduling model for reduced cpu energy," in Proc. 36th Annu. Symp. Foundations Comput. Sci., Oct. 1995, pp. 374-382.

[13] H.-S. Yun and J. Kim, "On energy-optimal voltage scheduling for fixepriority hard real-time systems," ACM Trans. Embedded Comput. Syst., vol. 2, no. 3, pp. 393-430, 2003.

[14] H. Aydin, R. Melhem, D. Mossé, and P. Mejia-Alvarez, "Power-aware scheduling for periodic real-time tasks," IEEE Trans. Comput., vol. 53, pp. 584-600, May 2003.

[15] V. Swaminathan and K. Chakrabarty, "Generalized network flow techniques for dynamic voltage scaling in hard real-time systems," in Proc. Int. Conf. Computer-Aided Design, Nov. 2003, pp. 21-25.

[16] L. H. Chandrasena, P. Chandrasena, and M. Liebelt, "An energy efficient rate selection algorithm for voltage quantized dynamic voltage scaling," in Proc. 14th Int. Symp. Syst. Synthesis, 2001, pp. 124-129.

[17] S. Lee and T. Sakurai, "Run-time voltage hopping for low-power realtime systems," in Proc. 37th Design Automation Conf., June 2000, pp. 806-809.

[18] W.-C. Kwon and T. Kim, "Optimal voltage allocation techniques for dynamically variable voltage processors," in Proc. Design Automation Conf., New York, June 2003, pp. 125-130.
[19] S. Saewong and R. Rajkumar, "Practical voltage-scaling for fixed-priority RT systems," in Proc. 9th IEEE Real-Time Embedded Technol. Applicat. Symp., May 2003, pp. 106-114.

[20] A. Manzak and C. Chakrabarti, "Variable voltage task scheduling algorithms for minimizing energy," in Proc. Int. Symp. Low-Power Electron. Design, Aug. 2001, pp. 9-14.

[21] I. Hong, G. Qu, M. Potkonjak, and M. B. Srivastava, "Synthesis techniques for low-power hard real-time systems on variable voltage processors," in Proc. 19th Real-Time Syst. Symp., Dec. 1998, pp. 178-187.

[22] N. AbouGhazaleh, D. Mossé, B. Childers, R. Melhem, and M. Craven, "Collaborative operating system and compiler power management for real-time applications," in Proc. 9th IEEE Real-Time Embedded Technol. Applicat. Symp., May 2003, pp. 133-141.

[23] C.-H. Hsu and U. Kremer, "The design, implementation, and evaluation of a compiler algorithm for CPU energy reduction," in Proc. ACM SIGPLAN Conf. Program. Lang. Design Implement., June 2003, pp. 38-48.

[24] Y. Zhang and K. Chakrabarty, "Task feasibility analysis and dynamic voltage scaling in fault-tolerant real-time embedded systems," in Proc. Design, Automation, Test Eur. Conf. Exhibit., vol. 2, 2004, pp. 21170-21175.

[25] A. Chandrakasan, S. Sheng, and R. W. Brodersen, Low-Power Digital CMOS Design. Norwell, MA: Kluwer, 1996.

[26] Technology Roadmap for Semiconductors (2003). Available: http://public.itrs.net/ [Online]

[27] Compaq IPAQ h3600 hardware design specification-Version $0.2 \mathrm{f}$ Available: http://www.handhelds.org/Compaq/iPAQH3600/iPAQ_ H3600.html [Online]

[28] B. C. Mochocki, "Voltage scheduling techniques for dynamic voltage scaling processors with practical limitations," M.S. thesis, Univ. Notre Dame, Notre Dame, IN, 2004.

[29] T. Ishihara and H. Yasurra, "Voltage scheduling problem for dynamically variable voltage processors," in Proc. Int. Symp. Low-Power Electron. Design, Aug. 1998, pp. 197-202.

[30] T. D. Burd, "Energy-efficient processor system design," Ph.D. dissertation, Univ. California, Berkeley, 2001

[31] N. Kim, M. Ryu, S. Hong, M. Saksena, C. H. Choi, and H. Shin, "Visual assessment of a real-time system design: A case study on a CNC controller," in Proc. 17th Real-Time Syst. Symp., Dec. 1996, pp. 300-310.

[32] C. D. Locke, D. R. Vogel, and T. J. Mesler, "Building a predictable avionics platform in ADA: A case study," in Proc. 12th Real-Time Syst. Symp., Dec. 1991, pp. 181-189.

\section{Probabilistic Crosstalk Delay Estimation for ASICs}

Kan Takeuchi, Kazumasa Yanagisawa, Takashi Sato, Kazuko Sakamoto, and Saburo Hojo

\begin{abstract}
The crosstalk delay caused by capacitive coupling between wires on a chip is investigated by using a statistical approach and circuit simulations. Two metrics are introduced in order to evaluate an impact of the crosstalk delay on timing design in advance. The first is probabilistic coupling rate (CPR), which can be obtained by the short segment model of the aggressors. Then, the CPR roughly obeys normal distribution and its standard deviation is determined by the slew time of the victim along with the number of aggressor segments. The second is crosstalk delay normalized by the original delay without crosstalk, $\Delta t_{\mathrm{pd}} / t_{\mathrm{pd}}$. The $\Delta t_{\mathrm{pd}} / t_{\mathrm{pd}}$ is equal to $2 * \mathrm{CPR}$ at the maximum, and CPR on average, regardless of victim length. The two metrics in conjunction with empirical slew distribution allows us to set the appropriate crosstalk delay budget, at the prelayout stage, for reducing the possibility of the crosstalk violation found in the postlayout verification process.
\end{abstract}

Index Terms-Crosstalk delay, probabilistic estimation.

Manuscript received July 18, 2003; revised October 23, 2003. This paper was recommended by Associate Editor F. N. Najm.

The authors are with Renesas Technology Corp., Tokyo 187-8588, Japan (e-mail: takeuchi.kan@ renesas.com).

Digital Object Identifier 10.1109/TCAD.2004.833605 